\title{
An Environment for Designing Exceptions in Workflows*
}

\author{
F. Casati, M.G. Fugini, I. Mirbel \\ \{casati,fugini,mirbel\}@elet.polimi.it \\ Politecnico di Milano \\ Piazza L. Da Vinci, 32 - I-20133 Milano, Italy
}

\begin{abstract}
When designing a WorkFlow schema, often exceptional situations, such as abnormal termination or suspension of task execution, have to be dealt with by the designer explicitly, as well as operations which typically occur at the beginning and termination of tasks. This paper shows how the designer can be supported by tools allowing him to capture exceptional behavior within a WF schema by reusing an available set of pre-configured exceptions skeletons. Exceptions are expressed by means of triggers, to be executed on the top of an active database environment; triggers can autonomously treat exceptional events or typical start/termination situations arising in a WF. In particular, the paper deals with the treatment of typical WF exceptional situations which are modeled as general exception skeletons to be included in a new WF schema by simply specializing or instantiating them. Such skeletons, called patterns, are stored in a catalog; the paper describes the catalog structure and its management tools constituting an integrated environment for pattern-based exception design and reuse.
\end{abstract}

\section{Introduction}

WorkFlow (WF) systems are currently becoming a more and more applied technology in the direction of providing a common framework for supporting activities within office information system.

WFs in the organizations are modeled to capture the possible sequences of activities needed to reach a given goal, and to provide tools and links to the existing Information Systems of the organization. Many aspects have to be considered in conjunction with the pure activity flow. In particular, the documents and information associated with the activities, the performers of the activities and their role in the organization, and the possible exceptions to the normal flow of activities. In other related fields, such as in Software Engineering and Information Systems, analogous techniques and tools are used combining data and function modeling to represent the application functionalities and to represent both normal and abnormal situations and exceptions[14].

Currently, few techniques and tools exist for WF design. Some of them [17] are based on rules and catalogs of predefined WF portions for WF management.

* Research presented in this paper is sponsored by the WIDE Esprit Project N. 20280 
In general, the designer of a WF schema has to provide many details in order to obtain a schema which is executable by a WorkFlow Management System (WFMS). This description requires the specification of both the normal flow and of the possible variations due to exceptional situations that can be anticipated and monitored.

This paper addresses the issue of providing support in designing exceptions in a WF focusing on exceptions which can be statically foreseen. It describes an environment of tools for the specification of WF schemas. Exceptions can be included in a schema by reusing (i.e., selecting and customizing) a set of pre-defined generic exceptions. The term "exception" is used in a broader sense than simply for exceptional situations. In fact, exceptions model also conditions regarding the starting, termination, or repetitions of WF instances (called cases) or regarding the periodical occurrence of operations in the WF execution.

The support environment, called WERDE, consists of a catalog of pre-designed WF schema portions, and of tools for accessing the catalog. We are mainly concerned with the specification of exceptional situations in WFs, that is, situations where events occuring in a WF schema may cause a task to be canceled, or a WF case to be terminated. Such exceptional situations may be designed in advance and inserted in the WF schema specification by including exception handlers for the most likely occurring events or by designing typical termination handling strategies. Another situation is the WF start phase which can be subject to typical situations. For instance, a WF has to be started periodically, or at a given time. These situations may be included as a pre-set part of the schema specification.

The catalog of the environment stores WF schema portions, mainly dealing with exceptions, to be reused during the design of a new schema. The contents of the catalog is a set of typical patterns[16] enabling the designer to deal with frequent occurrences of similar situations in WF design in given application domains, and with generic situations that may occur in any application domain. The catalog, and its related design support tools, is based on the following main issues:

- rules: rules model exceptions, starting and termination situations, and WF schema evolution due, for instance, to changed requirements deriving from new types of exceptional events;

- patterns: typical rules may be used in several designs. Patterns described in the paper are rules, or sets of related rules, to control a given typical situation. Patterns are a way for the designer to specify the WF schema by reusing previous design experience.

Rules in WF modeling have been considered, among others, by [12]. However, they are mainly used to represent the complete flow. In other approaches to WF modeling $([13,15])$, a strong emphasis is put on providing a simple and graphic interface to WF design. However, if such interfaces are to capture all the details of anomalous flows, readability is penalized, such as in Petri Net based approaches $([2])$. 
The approach of this paper based on pattern reuse aims at improving the speed and quality of WF schema design. A pattern is a generalized description of a rule or sets of rules that can be associated to a WF schema, either to model exceptions, or to model starting and termination situations, or situations to be controlled due to schema evolution. Reuse, also based on patterns, is beeing successfully applied to software development $([5,18,19])$.

The paper focuses on the functionalities of the WERDE environment which allow the designer to reuse patterns stored in a catalog for dealing with exceptions in WF schema design. Functionalities for pattern design are also presented. The WERDE environment is presented mainly in terms of specifications of its functionalities; then, the implemented functionalities are presented in detail.

The paper is organized as follows. In Section 2, we illustrate the catalog of patterns, and describe the use of rules in WF design by presenting exceptions and patterns. In Section 3, we present the functionalities of the environment for access and management of the catalog and show the tools usage for exception design in WF schemas and for exception analysis. Finally, in Section 4, we comment and give some hints about future work.

\section{A Pattern Catalog}

This section describes the pattern catalog of the WERDE environment where a set of pre-defined WF patterns is stored at different levels of abstraction and genericity in a layered structure. The catalog is the centralized repository of reusable WF elements in order to support specification by example of different elements of a WF, at different granularity levels, such as process variables, exceptions, and tasks. Exceptions and patterns will be described in the following of this section.

Patterns model situations that can occur in WF design in an applicationindependent way, at different levels of genericity (e.g., integrity constraints holding for schemas of any application domain, or schema fragments describing frequently occurring combinations of tasks in a given application domain). They can be described either using a language for specifying exceptions and/or through the WF process description language WPDL [6].

In Figure 1 the categories of patterns stored in the catalog are depicted. Patterns are classified at three different layers.

- Built-in patterns. Patterns at this level deal with reaction to events and time alarms (Basic patterns), integrity and security constraints over WF data (Authorization, Integrity, and Count patterns), WF management (Starting, Interaction, and Continuation patterns), and with changes in the organization structure (Organization and Schema evolution patterns). They describe context-dependent and time-dependent knowledge related to basic exceptional situations independent of the semantics of all possible applications. Built-in patterns specify, for example, basic interactions between WF elements, or situations where a task has to be suspended or resumed, or express 


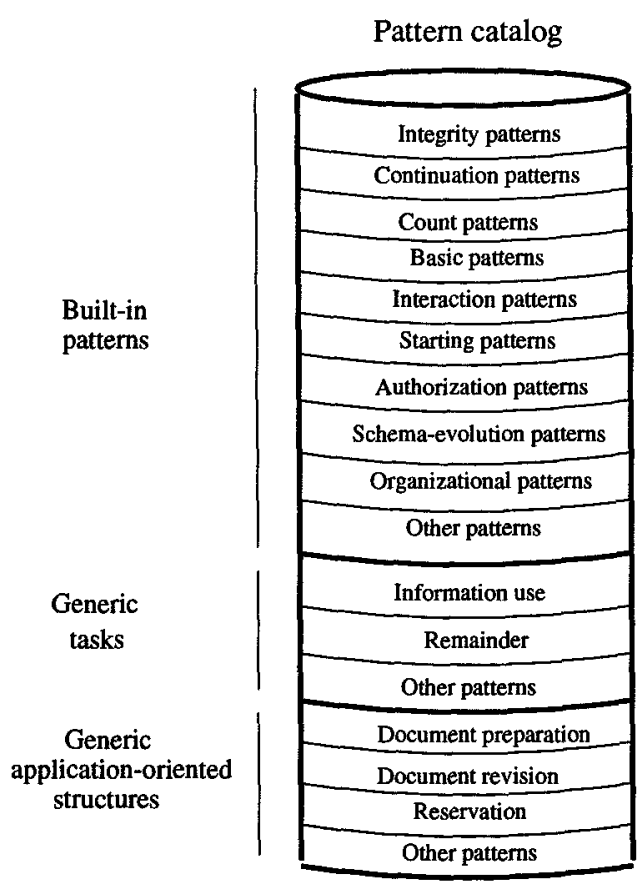

Fig. 1. The WERDE pattern catalog

exception requirements related to WF interoperability. Built-in patterns are implemented as generic rules that can be adapted to different application contexts.

- Generic tasks. Patterns at the generic tasks layer allow the description of frequently occurring activities in WF design in the form of tasks, independent of the documents involved in their execution (e.g., a task for registering information in a document).

- Generic application-oriented structures. This layer of the catalog contains WF fragments and associated exceptions needed to model frequently occurring situations of one or more application domains. These are frequently occurring combinations of tasks (i.e., WF fragments) generally performed together to achieve an organization goal in a given application domain (e.g., a generic structure describing the concurrent review of a document by several people).

In order to be accessible and user friendly for the designer, the catalog contains both a Pattern Specification element and a Sample Usage element. The first one describes the exception and includes parts allowing the designer to understand the goal of the pattern, to locate the pattern in the catalog, and to link patterns together within a WF schema. The second element contains sample instantiations of patterns in various application domains, thus guiding the designer in completing a new schema. 
In the following of the paper, we describe exceptions and patterns. In particular, we show how exceptions are specified and used, and how exceptions are then abstracted in patterns. For details about the patterns contained in the various categories of the catalog, the interested reader can refer to [9].

\subsection{Exceptions}

A short introduction to the exception language is now given (see [7] for a detailed description). The Chimera-Exc exception language is based on the Chimera language for active object-oriented databases. An exception is specified by an eventcondition-action (ECA) rule (also called trigger in the following). Events denote the occurrence of a possibly exceptional situation; conditions determine if the occurred event actually corresponds to an exceptional situation to be managed, while the action part defines the operations to be performed in order to manage the exception. ECA rules may refer in their event, condition, or action part, to objects and classes of the WFMS database. These include static and dynamic information on WFs and tasks (stored in objects of classes case and task respectively), data relating WF participants (objects of class agent), plus a WFspecific class, bearing the name of the WF, whose objects store process-specific variables local to each single case. For instance, variables needed for the execution of cases of a hotel roomReservation WF, such as the room number or the customer name, are stored as attributes of an object of the roomReservation class.

An exception can be sensitive to different kinds of events: data events are raised upon modification of the content of the WFMS database. Data events include the creation of a new object (create), the modification of an object's attribute (modify), and the deletion of an object (delete). For instance, event modify (task) is raised when an attribute of an object of the task class is modified. Workflow events are raised when a case or task is started or completed. WF events include caseStart, caseEnd, taskStart, and taskEnd. For instance, event taskStart (myTask) is raised when an instance of task myTask is started. Temporal events are raised upon the occurrence of a defined temporal instant. They can be expressed as deadlines, temporal periods, or interval elapsed since a specific date or time. For instance, event elapsed 1 day since taskStart (myTask) is raised as one day has elapsed since the start of an instance of task myTask. Finally, external events, qualified by their name, are notified by external applications: these may correspond to applicative situations, such as a phone call by a customer cancelling a reserved room. An example of external event is raise (cancelReservation).

The condition part consists of a declarative query, expressed by a formula evaluated over the WFMS database state. The formula is expressed as a conjunction of predicates, and includes a set of variables to which bindings are associated as a result of the formula evaluation: if the result of the query is empty (i.e., if no bindings are produced), then the condition is not satisfied, and the action part is not executed. Bindings resulting from the formula evaluation are passed to the action part in order to perform the reaction over the appropriate objects. 
The condition includes class formulas, for declaring variables ranging over the objects of a specific class (e.g., task $(T)$ ), type formulas for declaring variables of a given type (e.g., real(R)), and formulas expressing comparisons between expressions (e.g., T . executor="Lisa"). Objects affected by events are captured in the condition part by the occurred predicate. Thus, for instance, in an exception triggered by the caseStart event, the binding with the started instance is obtained by means of the predicates case(C), occurred (caseStart,C).

The action part includes data manipulation actions, updating the content of the WFMS database, and operations executed through calls to the WF engine. The first category includes the primitives create, delete, and modify. For instance, delete $(\log , L)$ deletes all objects of class Log to which $L$ is bound after the condition evaluation. The second category includes primitives to start, rollback, or suspend the execution of cases and tasks, assign or delegate the execution of tasks, and send messages to agents. Examples are delegateTask ( $T, A)$, startCase (mySchema, startingParameter), and notify (T.executor, "deadline is approaching").

A sample trigger activated at the end of cases of the roomReservation WF is as follows. The condition determines which is the case that has caused the triggering (predicates case(C), occurred (caseEnd,C)), retrieves the object storing the variables of the just completed case (roomReservation(R), R.case Id=C) and then checks if the customer has not paid (R.paymentDone=FALSE). If the condition is verified (i.e., if bindings are produced), then the action part is executed, causing the start of an instance of the cancelBooking WF and providing the customerID as input parameter.

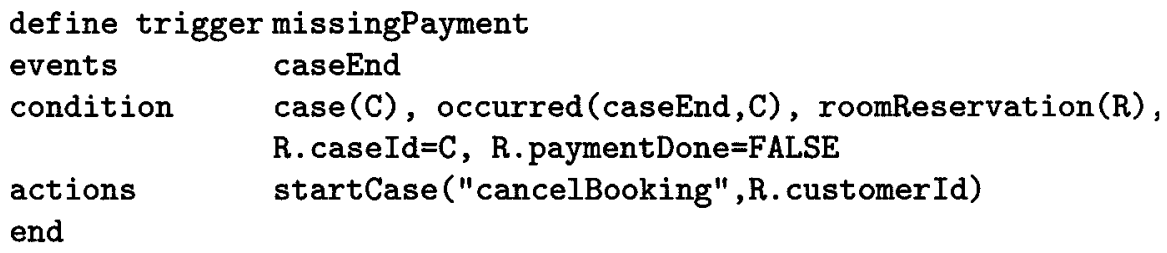

Rules can be associated to a task, to a schema, or to the whole WFMS. Tasklevel rules model exceptions which are related with a single task; an example is a rule reacting to the task deadline expiration. WF-level rules defines a behavior common to every task in a schema, or model exceptions related to the whole schema; for instance, a WF-level rule could specify that all tasks of a the WF should be suspended at 7pm. Finally, global (WFMS-level) rules model behaviors defined for every task or case, or exceptions involving cases of different WFs. For instance, an exception stating that "tasks that were assigned to an agent who left or is on vacation must be reassigned" should be declared at the WFMS-level, since it defines a general policy valid for every task.

Rules can be ordered by absolute priority. Upon concurrent trigger, the scheduler executes first the rules with higher priority. Among rules with the same priority, the choice is non-deterministic. 


\subsection{Patterns}

Since the Chimera-Exc language is quite flexible, the definition of a variety of exceptional behaviors is allowed. However, experience in exceptions design has shown that, although all the language features are required, most triggers follow common, repetitive "patterns". These observations led to the idea of taking advantage of repetitive patterns in triggers definition, in order to reduce the design effort and to provide guidelines for pattern reuse.

We introduce patterns as generalized descriptions of triggers and exceptional situations that can frequently arise in WF modeling. Patterns predefine typical rules or set of rules that capture the knowledge about the occurrence of an exceptional situation and the actions that can be performed to deal with it. Patterns consist of predefined parts, parameterized parts, and optional parts. Parameterization and optionality are introduced to support pattern re-usability and adaptation in WF design for the aspects related to exception modeling and handling.

Pattern Description: The pattern are mainly described by a specification part and a sample usage as follows.

Specification part: The specification part of the pattern is the description of an exception or of an application, including a set of textual fields and a WPDL and/or Chimera-Exc portion implementing the pattern. In particular, the pattern specification is structured in the following fields:

- name, uniquely identifying the pattern in the catalog of all patterns;

- intent, a textual part describing the purpose of the pattern (intended scope and known uses);

- classification, according to the categories and sub-categories of the catalog;

- template, containing the core specification of the pattern. It can be provided in two forms:

- For patterns representing exceptions, the template contains the specification in terms of events, conditions, and actions. On the contrary of the events and conditions, which are the main parts of the patterns, the action part provides only suggestions. This reflect the fact than exception patterns focus on how to capture exceptions, more than on how to fix reactions, which are application dependent: in a given application, a warning message can be a satisfying reaction, when a cancelation of the case can be more suitable in another application of the same pattern.

- For patterns representing application structures, the template contains the diagrammatic WF schema skeleton describing the application fragment.

The template usually contains parametric fields to be filled in with specific values provided by the user; mandatory and optional parts can also be specified. 
- keywords, which are a set of user-selected terms that can be used to refer (select, search, etc.) to the available patterns in the catalog; this field allows one to describe more precisely the topics of the pattern, especially to distinguish the different patterns of a given category in the classification;

- related to, establishing links among patterns to combine them in different structures;

- guidelines, providing suggestions to the user about possible usage and personalization of patterns.

In Figure 2, an example of pattern specification is given. It is a pattern to be used to start a new case upon termination of another one.

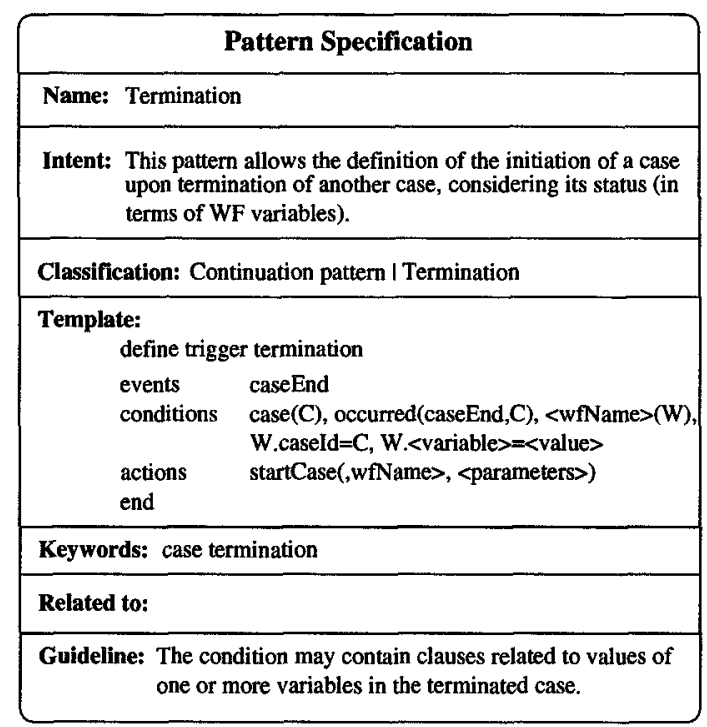

Fig. 2. The Termination pattern

The complete description of the pattern-specification language can be found in [8].

Sample usage part: Since the user of the catalog is intended to be an expert of the application under design and is not required to have a detailed knowledge of the Chimera-Exc syntax, the pattern model is endowed with the user oriented sample usage pattern. This is a set of instantiations of patterns on specific examples. They show how patterns can be personalized in different contexts and applications by illustrating how parameters of patterns can be supplied by the designer to produce a concrete WF. The sample usage part of the pattern description is a set of WF-specific instantiations of patterns related to an application domain. In general, several examples are provided for a pattern for different domains. The sample usage is an instantiation of the variables/parameters appearing in 
the template field of the pattern specification according to the domain selected to construct the example. The missingPayment trigger given in Sec. 2.1 is a sample usage of the pattern termination presented in Figure 2.

Pattern Reuse: Two basic mechanisms are provided for reusing patterns in WF design: specialization and instantiation. Pattern specialization is a mechanism for creating a new pattern starting from an existing one. Pattern instantiation is a mechanism for creating triggers for a particular situation by using a pattern.

Pattern specialization: When designing triggers, one may want to specialize a trigger previously defined. Since it was already explained, the action part of the trigger provides only suggestions, on the contrary of the events and conditions parts. Therefore, the specialization focuses on the event and condition parts. Three ways are given to specialize a trigger. They can be combined.

- The first way consists in rewriting the trigger using more specific genericterms. Consider a pattern composed of a trigger with an event part containing the generic term <referenceEvent>. If one wants to write another pattern, with the same structure as the previous one, but focused on external events only, one can specialize the exception part of the pattern by replacing 〈referenceEvent $>$ with the more specialized generic-term 〈externalEvent>.

- The second way consists in instantiating part of the trigger. This is done by replacing a generic term by a non-generic one. For example, <class $\rangle$ in a given trigger of a pattern can be specialized into task in a more specific pattern dealing only with tasks.

- The third way consists in adding conditions and actions to a given trigger in order to obtain a more specialized one. These additions can be done only in the condition and actions parts. Adding events is not allowed since this would change the invocation of the trigger.

Pattern instantiation: Patterns are used when designing a new WF as follows. After choosing the suitable pattern, this has to be instantiated. In general, this is done by replacing the generic-term with a value, for example 〈referenceEvent $\rangle$ becomes documentArrival. A more specific name must also be assigned to the pattern and the triggers. If there are optional parts, the user has to decide if he/she takes the optional parts or not. If several possible actions are defined in the action part, it is also necessary to choose one or several of these actions.

It is also allowed to add conditions or actions to the trigger, for example to test the value of a variable which is specific to the WF under design.

A more complete and formal description of the instantiation and specialization mechanisms can be found in [8].

\section{Specification of the Exception Design Environment}

In this section, we present the environment supporting the pattern-based approach illustrated in Section 2. The tool is mainly given in terms of specification of its functionalities which deal with pattern management in the catalog 
and with the design and analysis of exceptions. The current prototype environment implementing most of the functionalities for catalog management, and for pattern-based exception design and analysis is finally described.

\subsection{Management of Catalog}

Management of the pattern catalog deals mainly with the insertion, modification and deletion of patterns. However, it also takes into account the management of the categories useful to classify and retrieve patterns. In this section, the environment functionalities dealing with management of categories are presented, followed by the functionalities for pattern management.

Category management: As presented in Section 2, the catalog is divided in categories (built-in patterns, generic tasks and generic-application oriented structures). Each is divided in sub-categories: Termination, for example is a sub-category of the built-in patterns category. Since the catalog evolves during time, it is possible to $a d d$ new categories and sub-categories, and to remove some of them. For removal, a check is performed to ensure that no pattern of the catalog refers to the category or sub-category being deleted.

Pattern management: This functionality deals with creation, modification and deletion of patterns.

When creating a pattern, its different parts have to been fill-in by the user: name, intent, classification, template, keywords, related-to patterns and guidelines. The pattern name must be unique. The classification conforms to the categories and sub-categories of the catalog. Patterns keywords can be selected from a tool-proposed list of keywords and belong to a thesaurus automatically updated whenever new keywords are specified for newly defined patterns. Guidelines can be textual information to be compiled off-line by the pattern designer. Links between templates ("related-to" fields) can be defined by activating an "add link" and starting a corresponding dialog window. Sample usages can also be written to facilitate pattern reuse. If the specialization mechanism is used to create a new pattern, an existing pattern $P$ can be selected, and the designer can select the parts of $P$ that must be modified. The pattern has to share at least one keyword of its generic pattern (the pattern from which it is derived) and has to be placed in the same category/sub-category. Links are maintained in the catalog between patterns belonging to a specialization chain.

When modifying patterns, in addition to the modification of its features (intent, template, keywords, related-to fields and guidelines), new sample usages can be added to an existing template, by defining an "executable example" and by selecting the template of interest from the catalog. It is also possible to change the category and/or sub-category of a pattern. This means changing the location of the pattern in the repository, but also changing the location of the patterns linked to it through specialization or generalization, in order to maintain the catalog consistency. 
Deletion of patterns is performed by selecting the pattern of interest. The tool then performs a set of basic integrity checks:

- if another pattern uses the current one, the link is also removed;

- if the pattern is involved in an inheritance hierarchy (is inherited by at least a pattern $p_{2}$, and possibly inherits also from at least pattern $p_{1}$ ), the user has to choose (i) to delete also $p_{2}$, or (ii) to build a new inheritance link between $p_{1}$ and $p_{2}$.

\subsection{Design of Exception}

The environment support the pattern-based approach to the design of exceptions in WF schemas limited to the design of its exceptions. In the following, the word exception schema will represent the set of exceptions related to a given WF schema.

Exceptions can be designed following a bottom-up process, based as much as possible on reusing the patterns available in the catalog through the instantiation mechanism. The tool guides the designer during the process of exception specification by providing both classification information, which facilitates the search in the catalog, and guidelines to enter values for pattern parameters and to compose patterns into more complex structures.

When creating an exception schema, exceptions can be written (created) from retrieved patterns. These patterns are instantiated/personalized for the WF schema at hand. New exceptions can also be defined from scratch, if the available patterns do not fit the WF requirements.

Two search modalities are provided, namely, keyword-based and classificationbased search. The first relies on a pre-defined thesaurus of terms loaded at pattern specification time to characterize the pattern within the catalog. Keywords can refer either to pattern elements (e.g., names of events, actions) or can be more generic, domain-related keywords. The second modality allows for the retrieval of patterns based on two orthogonal views of the catalog:

- typology of pattern, with respect to event types (e.g., temporal, external, data);

- abstraction level of patterns in the catalog (built-in, generic, applicationoriented).

Patterns matching search criteria are provided and can be manipulated by the designer.

The instantiation consists in binding the pattern parameters on the basis of the semantics of the WF schema, by exploiting the syntax-driven capabilities of the tool and its interface facilities. A set of dialog windows drives the designer in entering the patterns fields, possibly enabling the visualization of the guidelines and usage examples associated with the template under manipulation. New conditions and actions can be added to the exceptions derived from the patterns. The tool dialog facilities support also the definition of new exceptions from scratch. Triggers within a given exception schema can be modified and/or deleted 
as needed by the designer. No special checks have to be performed, because the triggers, in this part of the tool, are not linked to the patterns of the catalog.

Exception schemas can be modified, with respect to their exceptions. These modifications include insertion of triggers, by searching in the catalog or by writing them from scratch, modification of the existing triggers, and deletion of some of them if they are no longer needed.

\subsection{Schema Analysis}

Exceptions in WFs are typically designed individually, by identifying the exceptional situation, selecting the appropriate pattern from the catalog, and instantiating it in order to obtain a Chimera-Exc exception. However, although an exception considered individually may be correctly specified, mutual interactions among exceptions may lead to unforeseen and undesired behaviors. For instance, a set of exceptions may trigger each other, or two exceptions might operate on the same data, with the consequence that, if they are concurrently triggered, the final result of their execution depends on their non-deterministic execution order.

Exceptions analysis aims at statically (i.e., at compile-time) verifying that such undesired interactions do not occur. In particular, it aims at verifying two significant properties for a set of exceptions: termination and confluence $[1,3,4$, 20]. Termination holds if exceptions do not trigger each other indefinitely, so that rule processing eventually terminates. Termination analysis is typically based on building graphs depicting triggerings and activations among exceptions, and on detecting cycles in these graphs (see $[4,7]$ for details).

Confluence requires the final effect of the rule processing (represented by direct or indirect changes to the WFMS database) to be independent on the (non-deterministic) scheduling of triggers having the same priority [8]. Sufficient conditions for confluence have been proposed in $[1,3,8]$. Unfortunately, the above properties are undecidable in the general case.

A fully automated analysis of interactions between exceptions is generally difficult to achieve, since it requires to understand the semantics of conditions and actions. A simple, conservative solution consists in checking that exceptions query and operate on different classes. More sophisticated and less conservative techniques for rule analysis, which consider the semantics of conditions and actions, have been proposed in [3].

In addition to adapting techniques proposed for active database rule analysis, a tool performing exception analysis may take into account the context in which exceptions are declared; in our model exceptions are always active, and if two exceptions share the same triggering event, they are both triggered as the event occurs, regardless of which part of the case is currently in execution. Exceptions can be concurrently triggered even if they are raised by different events, since exception scheduling occurs periodically, and within two subsequent exception processings many events can occur and many exceptions can be triggered, making the analysis more and more complex. However, although exceptions are always 
triggerable regardless of the context where they are declared, it is a good design practice to constrain the exception (within the condition part) to be active only when the element in which they are declared (e.g., a task) is running. If this is done, then we can reduce the scope of the analysis to exceptions of tasks that may be concurrently active (besides WF- and WFMS-level exceptions), thus reducing the number of exceptions that need to be concurrently analyzed. This is a key issue in exception analysis, since sufficient conditions for termination and confluence are rarely met when the analysis is extended to the globality of declared exceptions, particularly when conservative approaches are followed.

\subsection{Tool Architecture}

This section presents the architecture and the functionalities provided by the WERDE tool and gives a usage example. The architecture is depicted in Figure 3.

The definition of an exception for a WF schema is performed through the Schema Editor module, based on reusing the available patterns. From within the Schema Editor, the designer can interactively search the catalog for a suitable generic pattern using the Exception Retriever module. Retrieved patterns are instantiated/personalized for the WF schema at hand, through the Exception Writer module, which can also be used to define new patterns or exceptions from scratch, if the available ones do not fit the WF requirements. Exceptions defined for a schema can be analyzed by exploiting the Schema Analyser module of WERDE. The Catalog Manager performs the catalog management functions that is, insertion, modification and deletion of patterns.

A Visual $\mathrm{C}++$ /Windows prototype of WERDE is available, operating in a Client/Server environment, in accordance with the design guidelines of the other WIDE tools [6].

Managing the Catalog: The pattern catalog is extensible by defining new patterns and/or specializing the existing ones. To this purpose, WERDE provides maintenance functionalities to manage the catalog (Catalog Manager module) by allowing insertion of new patterns and modification or deletion of existing ones. It also allows to add/remove pattern categories and sub-categories.

\section{Pattern management:}

- Creation: Newly defined patterns are stored in the catalog, under the proper category/sub-category. Fields of the pattern specification are interactively filled in. The pattern template is specified by invoking the Exception Writer editor. Patterns keywords can be selected from a proposed list. Keywords in the thesaurus are automatically updated whenever new keywords are specified for newly defined patterns. Guidelines can be inserted as textual information to be compiled off-line by the pattern designer. 


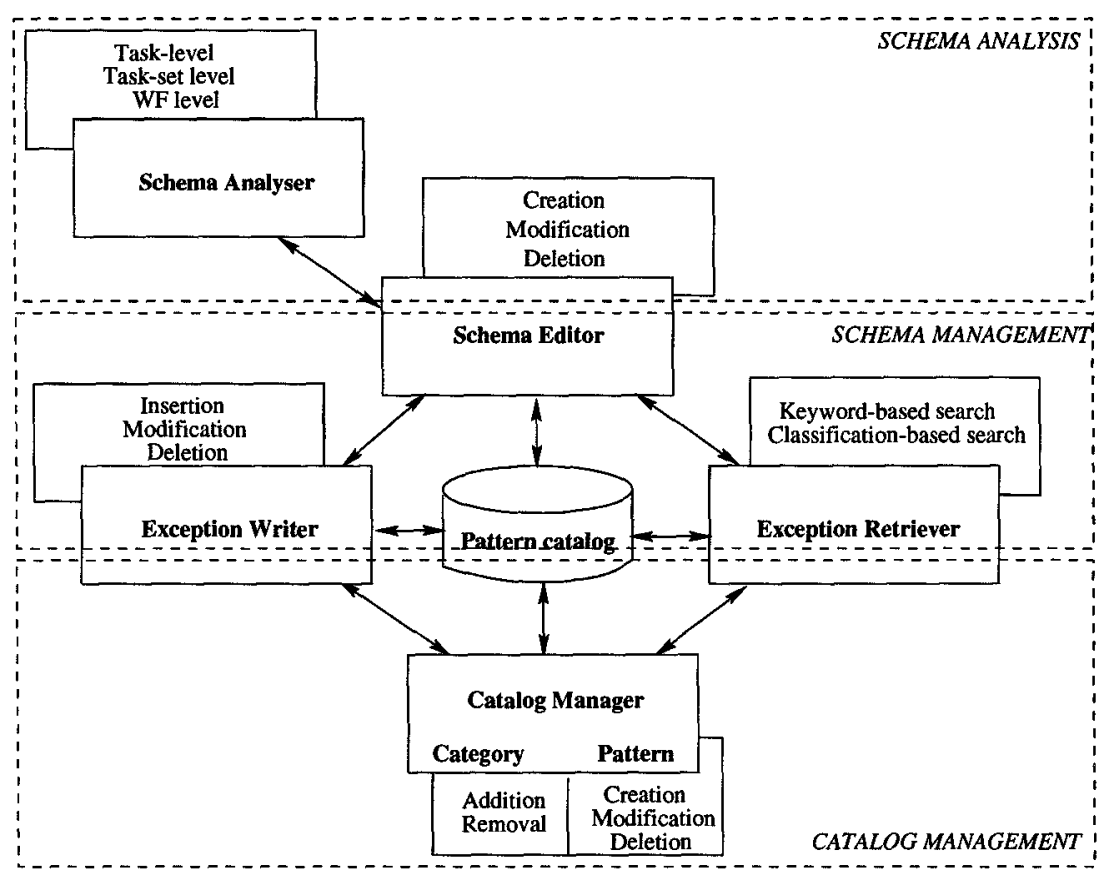

Fig. 3. Architecture of the WERDE tool

- Modification: An existing pattern can be modified, after being selected using the Exception Retriever. The designer can then select the parts of the pattern that must be modified, and operate on them through dialog windows. In WERDE it is possible to add new sample usages to an existing pattern template, by defining an "executable example" and by selecting the template of interest from the catalog.

- Deletion: it is performed by selecting the pattern of interest, and the tool performs the basic integrity checks.

An example of work session, dealing with the specification of the termination pattern shown in Figure 2, is presented in Figure 4.

Insertion/removal of categories: Categories and sub-categories can be added to the catalog at each level. Removal of categories implies the deletion of all subcategories, provided that the designer has already cancelled all the corresponding patterns.

Using the Catalog: WERDE guides the designer during the process of exception specification by providing both classification information, which facilitates the search in the catalog, and guidelines to enter values for pattern parameters and to compose patterns into more complex structures. 


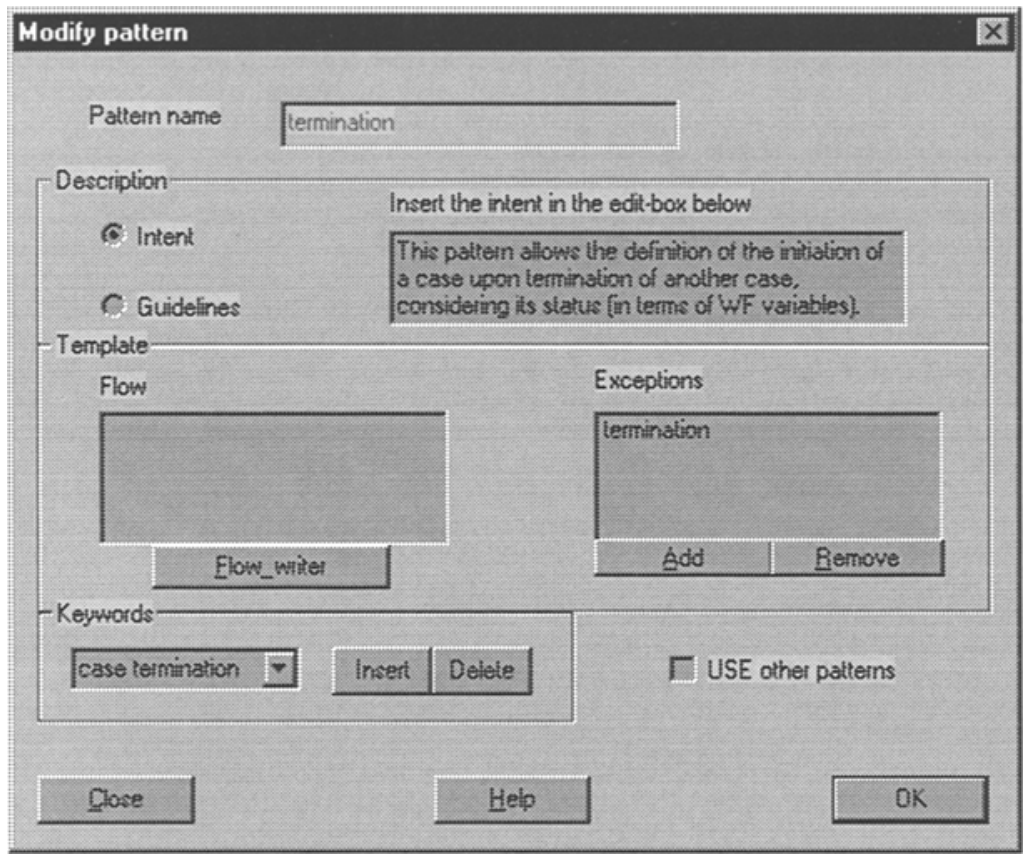

Fig. 4. Creation of the termination pattern in WERDE

A typical exception-design session consists in activating the Schema Editor module. The main functionalities that can be invoked from within this module deal with the creation, modification and deletion of exception schemas. The functionalities provided through the creation and modification of exception schemas are the following:

- Retrieval, to access the catalog and search for suitable patterns. The two search modalities presented above are implemented (keyword-based and classification-based search).

Figure 5a shows the "Retrieve Option" screen where the various search modes are available. In particular, consider that the termination pattern is being searched by keywords, as depicted in Figure 5b. The result of the search appears in Figure 5c.

- Insertion, to support the interactive definition of exceptions starting from a retrieved pattern or from scratch. The instantiation consists in binding the pattern parameters on the basis of the semantics of the WF schema, by exploiting the syntax-driven capabilities of the Exception Writer module and the template interface facility of WERDE. This facility consists of a set 


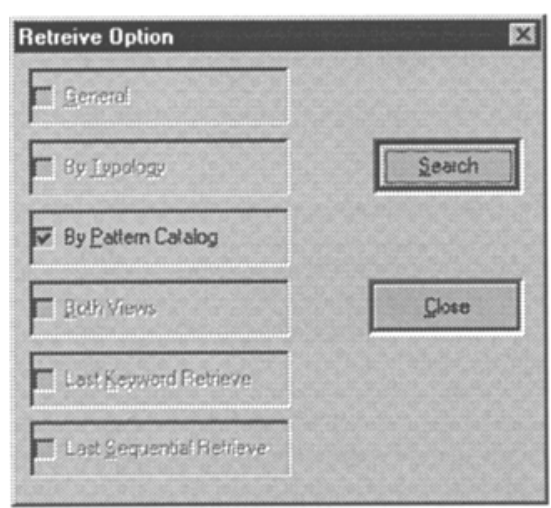

(a)

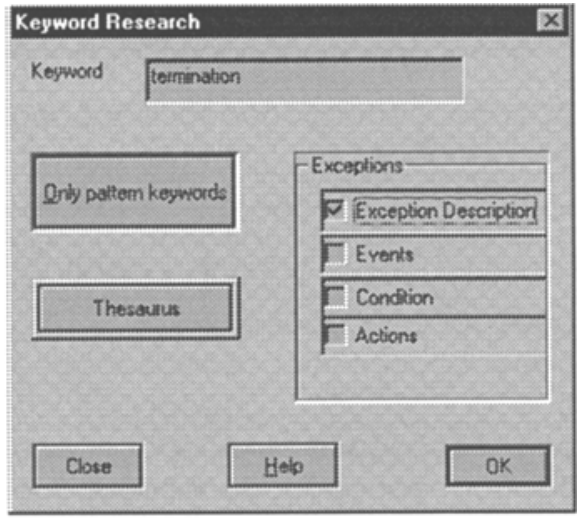

(b)

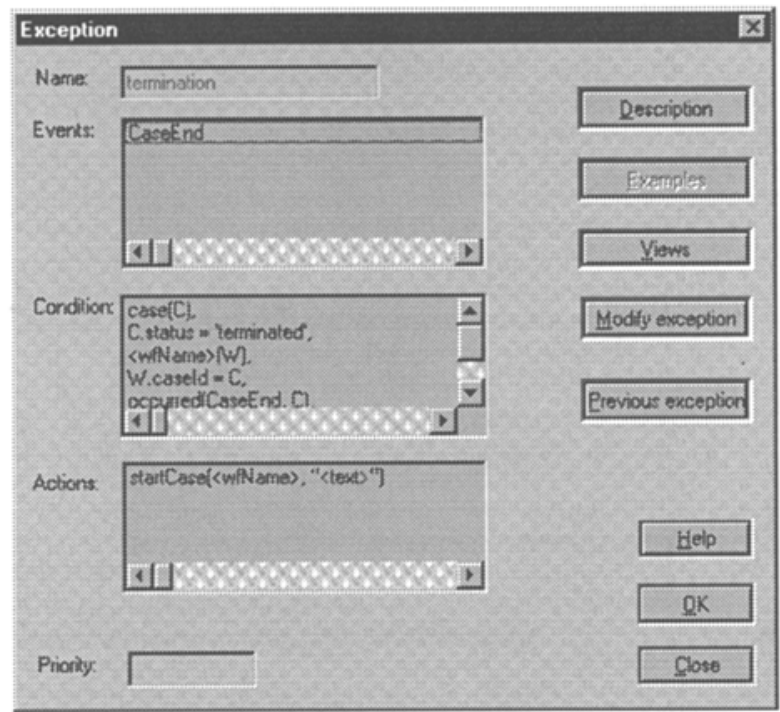

(c)

Fig. 5. Pattern retrieval. Selection of retrieval option (a), keyword specification for keyword-based retrieval (b), and presentation of the retrieved pattern (c) 
of dialog windows which drives the designer in entering the patterns fields, possibly enabling the visualization of the guidelines and usage examples associated with the template under manipulation. The dialog facilities of the Exception Writer support also the definition of new exceptions from scratch. The instantiation of the termination pattern retrieved through the steps depicted in Figure 5 works as in the sample work session of Figure 6, where the pattern is instantiated to obtain the trigger missingPayment of section 2.1. The instantiation steps consist of activating the exception writer module, selecting the item to be instantiated (<wfName>W in the example of Figure 6), and overriding it with the desired Chimera-Exc condition (roomReservation(R)).

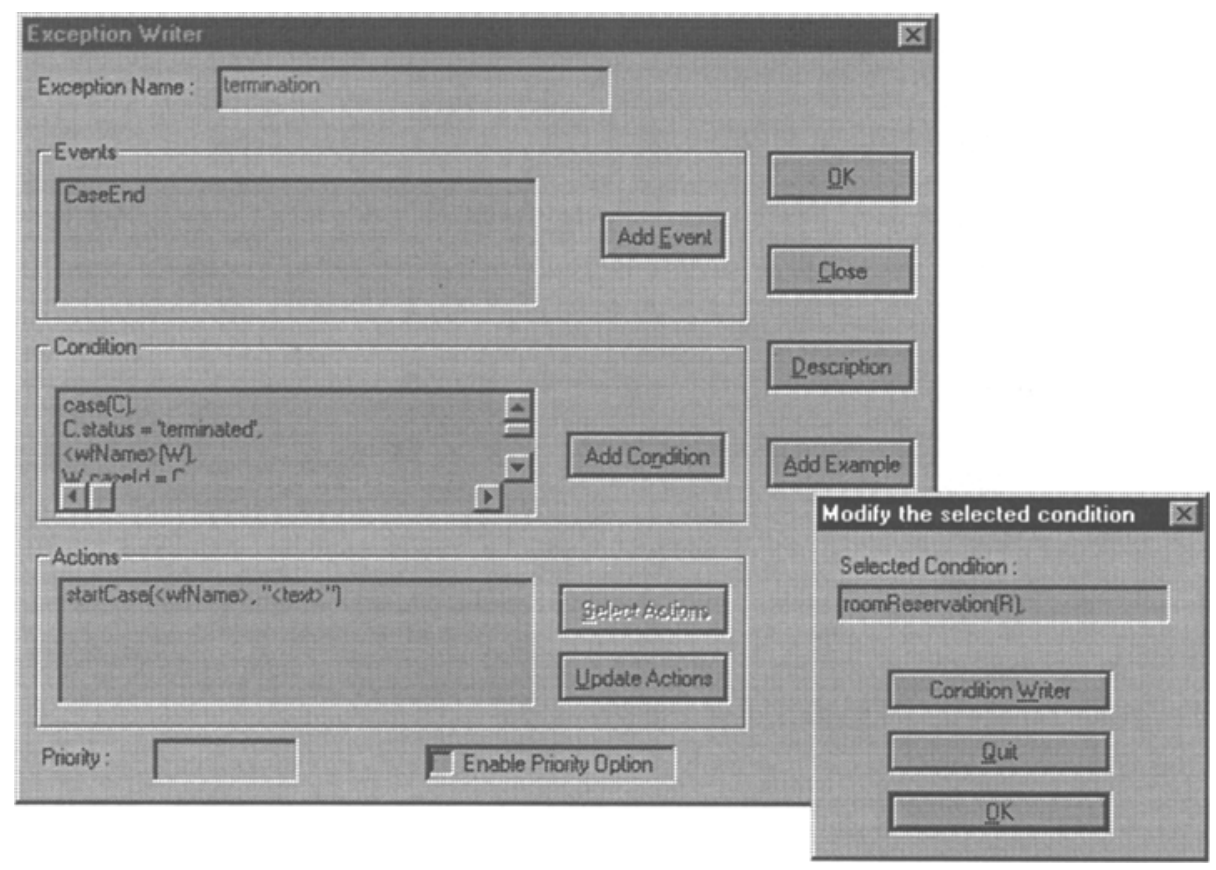

Fig. 6. Instantiation of the termination pattern

- Modification, to modify exceptions inside exception schemas.

- Deletion, to remove exceptions from exception schemas.

- Analyze, to analyze the exceptions written for the current schema, using the Schema Analyser module. WERDE currently provides a basic set of the static analysis functionalities, by focusing on interactions within each pair 
of exceptions. In WERDE, two triggers are considered as conflicting if they are active simultaneously and contain incompatible actions; actions are incompatible if the order of their execution leads to different states of the WF management database [6]. A table of incompatible actions is maintained to support the analysis, which can be performed at three levels:

- task level, to check exceptions defined for a given task;

- task set level, to select a number of tasks for which the exceptions must be analyzed;

- schema level, to check all the exceptions defined in the schema.

Anomalous situations detected by the Analyser are signaled to the designer who can activate the Exception Writer for editing the involved exceptions.

\section{Concluding Remarks}

We have presented the WERDE environment supporting the design of exceptions in WF schemas. The environment is based on a catalog of WF patterns which are a generalized description of triggers which can be reused when designing a new WF schema. Exceptions model both abnormal situations and typical start/end/repetitions of operations in a WF. We have presented the structure of the pattern catalog and the mechanisms for pattern reuse. We have also described the related functionalities of the WERDE tools for exception design and catalog management.

Currently, the catalog is populated with a set of patterns which have been constructed by analyzing a set of real cases provided in the WIDE project testbed. A set of primitives for pattern reuse has been fully specified in [8] and is being tested to check the quality of these patterns in terms of significance and accessibility for reuse. Particularly the sample usages of patterns stored in the catalog are proving to be an effective means for guiding the designer in selecting the appropriate patterns, in instantiating them appropriately, and in inserting the exceptions correctly in the WF schema.

The implementation of WERDE has currently completed most of the functionalities described in the paper. Some functionalities, such as pattern design and schema analysis, are being extended in order to achieve a more complete and effective suite of functions. For example, for schema analysis, we plan to consider exception termination analysis and confluence analysis on rule sets, and to consider in more detail the semantics of actions and conditions. For pattern design, we plan to have functionalities which help the catalog administrator in abstracting exception skeletons from WF schemas in a guided way and storing new patterns in the appropriate categories with suitable links to the existing repository. In fact, the catalog is regarded as an extensible tool to be populated gradually with new categories of patterns to be defined by examining further real WF applications. Further points of research regard the use of exceptions as a basis for WF dynamic evolution and the design of a user feedback mechanism for validation of the quality and effectiveness of a given WF, using adaptive techniques such as those studied in [11]. 
Acknowledgments: The authors are thankful to B. Pernici and S. Castano for common work and ideas and to the students who implemented the tool.

\section{References}

1. A.Aiken, J.Widom, J.Hellerstein. "Behavior of database production rules: termination, confluence, and observable determinism". Proceedings of SIGMOD, San Diego, CA, 1992.

2. S. Bandinelli, A. Fuggetta, C. Ghezzi, "Software process model evolution in the SPADE environment", IEEE Transactions on Software Engineering, December 1993.

3. E. Baralis, J. Widom, "An algebraic approach to rule analysis in expert database systems", Proceedings of VLDB 94.

4. E.Baralis, S.Ceri, S.Paraboschi. "Improving rule analysis by means of triggering and activation graphs", Proceedings of the 2nd International Workshop of Rules in Database Systems, Athens, Greece, 1995.

5. R. Bellinzona, M.G. Fugini, B. Pernici, "Reusing specifications in OO applications", IEEE Software, vol. 12, n. 2, March 1995.

6. F. Casati, P. Grefen, B. Pernici, G. Pozzi, G. Sanchez, "WIDE workflow model and architecture", Technical Report, Politecnico di Milano, n. 96-050, 1996.

7. F. Casati, S. Ceri, B. Pernici, G. Pozzi, "Specification of the rule language and active engine of FORO v.1", WIDE Technical Report n. 3008-6, September 1997.

8. F. Casati, S. Castano, M.G. Fugini, I. Mirbel, B. Pernici. "Using patterns to design rules in workflows", Technical Report n. 97-065 of the Politecnico di Milano, November 1997.

9. S. Castano, M.G. Fugini, I. Mirbel, B. Pernici, "Workflow reference models", WIDE Technical Report n. 3015-2, June 1997.

10. S. Ceri, R. Ramakrishnan, "Rules in database systems", ACM Computing Surveys, Vol. 28, No. 1, March 1996.

11. E.Damiani, M.G. Fugini, E. Fusaschi, "COOR: a Descriptor Based Approach to Object-Oriented Code Reuse", IEEE Computer Magazine, September 1997.

12. U. Dayal, M. Hsu, R. Ladin, "Organizing long-running activities with triggers and transactions", Proceedings of ACM SIGMOD, 1990.

13. D. Georgakopoulos, M. Hornick, A. Sheth, "An overview of workflow management: from process modeling to workflow automation infrastructure, distributed and parallel databases", Vol. 3, n. 2, April 1995.

14. C. Ghezzi, M. Jazayeri, D. Mandrioli, "Fundamentals of software engineering", Prentice-Hall Intl., 1991.

15. D. Hollingsworth, "Workflow management coalition, the workflow reference model", Technical Report n. TC00-1003, November 1994.

16. R.E. Johnson, "Frameworks = components + patterns", in Communications of the ACM, vol. 40, n. 10, October 1997

17. G. Kappel, P. Lang, S. Rausch-Schott, W. Retschitzegger, "Workflow management based on objects, rules, and roles", IEEE Data Engineering Bulletin. Special issue on WF Systems, vol. 18, no. 1, March 1995.

18. C.W. Krueger, "Software reuse", ACM Computing Surveys, vol.24, n.2, June 1992.

19. D.C. Rine, "Supporting reuse with object technology", IEEE Computer, Special Issue on OO Development and Reuse, October 1997.

20. J. Widom, S. Ceri, "Active database systems: triggers and rules for advanced data processing", Morgan Kaufmann, San Mateo, CA, 1996. 